\title{
Upregulation of FTX expression is associated with a poor prognosis and contributes to the progression of thyroid cancer
}

\author{
PING WANG $^{1}$, YONGMING ZHANG $^{1}$, WENPING WANG $^{1}$ and HUIMIN JIANG ${ }^{2}$ \\ Departments of ${ }^{1}$ Endocrinology and ${ }^{2}$ Hematology, Anhui No. 2 Provincial People's Hospital, Hefei, Anhui 230041, P.R. China
}

Received Dcember 8, 2020; Accepted April 23, 2021

DOI: 10.3892/ol.2021.12933

\begin{abstract}
The dysregulated expression of long non-coding RNA FTX transcript X inactive specific transcript regulator (FTX) has been reported to be involved in the tumorigenesis of multiple cancer types. However, to the best our knowledge, its function and clinical value in thyroid cancer remain unclear. The present study aimed to determine the potential role of FTX in the development and progression of thyroid cancer. Reverse transcription-quantitative PCR analysis revealed that the expression levels of FTX were upregulated in thyroid cancer tissues and cell lines compared with those in normal tissues and cell lines, respectively. Survival analysis demonstrated that patients with upregulated FTX expression had a lower survival rate. Functional experiments revealed that the knockdown of FTX inhibited proliferation, cell cycle progression, migration and invasion, and induced apoptosis in thyroid cancer cells, while FTX overexpression accelerated proliferation, migration and invasion, and alleviated apoptosis in thyroid cancer cells. In addition, FTX knockdown significantly inhibited tumor growth in vivo. Furthermore, in thyroid cancer cells, FTX was identified to positively regulate the expression levels of TGF- $\beta 1$, which is known to play an important regulatory role in tumor metastasis. In conclusion, the findings of the present study suggested that FTX may accelerate thyroid cancer progression via regulation of cellular activities, including cell proliferation, migration, invasion and apoptosis. Thus, FTX may represent a potential biomarker for the diagnosis, treatment and prognosis of thyroid cancer.
\end{abstract}

\section{Introduction}

Thyroid cancer is a leading cause of mortality among endocrine tumors (1). In total, 562,000 individuals were diagnosed with thyroid cancer worldwide in 2018 (2). Although significant

Correspondence to: Dr Huimin Jiang, Department of Hematology, Anhui No. 2 Provincial People's Hospital, 1868 Dangshan Road, North Second Ring, Hefei, Anhui 230041, P.R. China

E-mail: huimin_j@126.com

Key words: FTX transcript X inactive specific transcript regulator, thyroid cancer advances have been made in the therapeutic strategies used to improve the long-term survival of patients with thyroid cancer, $\sim 20 \%$ of patients with thyroid cancer experience recurrence and distant metastasis within 10 years $(3,4)$. Since thyroid cancer usually progresses slowly, it has no obvious different clinical manifestations from benign thyroid diseases, which often leads to misdiagnosis and untimely treatment (5). Nowadays, increasing numbers of molecular biomarkers have been identified as diagnostic and therapeutic biomarkers in cancer, which can improve the early detection of cancer and decreased the mortality rate $(6,7)$. For example, mircroRNA-203 (miR-203) promoted estrogen receptor-positive breast cancer growth and stemness, and may be used a therapeutic target for breast cancer (8). In addition, overexpression of c-Fos enhanced tumor growth in vivo and the stemness in head and neck squamous cell carcinoma (HNSCC) cells, and this may hold potential as a cancer stem-like cell-directed therapeutic approach to improve HNSCC treatment (9). Therefore, it remains an urgent requirement to determine novel sensitive and specific biomarkers for the diagnosis, clinical treatment and prognosis of thyroid cancer.

Long non-coding RNAs (lncRNAs) are >200 nucleotides in length and lack protein coding ability. It has been widely reported that the dysregulated expression of IncRNA is associated with multiple biological functions and participates in the development of multiple cancer types, including thyroid cancer. For instance, a previous study reported that the upregulated expression levels of lncRNA X inactive specific transcript (XIST) accelerated cell proliferation and tumor growth in thyroid cancer (10). The overexpression of lncRNA metastasis-associated lung adenocarcinoma transcript 1 contributed to the angiogenic process of thyroid cancer via the regulation of fibroblast growth factor 2 secretion (11). Furthermore, lncRNA n340790 promoted thyroid cancer tumorigenesis by modulating miR-1254 expression (12). Thus, to determine potential novel therapeutic treatments for thyroid cancer, it remains important to identify novel tumorassociated lncRNAs and to determine their biological role and mechanisms of action.

The lncRNA FTX transcript XIST regulator (FTX), located in the XIST gene locus, has been reported to act as a tumor promoter in various types of cancer, including glioma, lung adenocarcinoma, colorectal cancer, osteosarcoma and gastric cancer, where it was found to be closely associated with a poor prognosis (13-17). In addition, the expression levels of 
FTX were found to be significantly upregulated in sporadic medullary thyroid cancer (18). However, to the best of our knowledge, the clinical characteristics, biological functions and underlying mechanism of FTX in thyroid cancer have yet to be determined.

The current study aimed to determine the expression levels of FTX in thyroid cancer and to elucidate the biological functions of FTX in thyroid cancer cell proliferation, migration, invasion and apoptosis. The results of the present study provide novel evidence of the potential role of FTX in the development and progression of thyroid cancer, which may provide a novel insight into future therapeutic directions for patients with thyroid cancer.

\section{Materials and methods}

Patient studies. A total of 58 thyroid cancer and adjacent normal tissues ( $3 \mathrm{~cm}$ away from the tumor) were obtained from patients (mean age, 51 years; age range, 41-62 years) during surgery at The Anhui No. 2 Provincial People's Hospital (Hefei, China) between March 2015 and January 2017. All patients enrolled in the present study had not received radiotherapy or chemotherapy prior to surgery. All samples were stored in liquid nitrogen before use. Each patient provided written informed consent prior to participation in the study. The patient experimental protocols were approved by The Ethical Committee of Anhui No. 2 Provincial People's Hospital.

Cell lines and culture. Thyroid cancer cell lines (FTC-236, SW-1736 and 8305C) and a normal human thyroid epithelial cell line (Nthy-ori3-1) were purchased from The Cell Bank of Type Culture Collection of The Chinese Academy of Sciences, and tested for mycoplasma and authenticated by STR profiling. Cells were cultured in DMEM supplemented with 10\% FBS (both Gibco; Thermo Fisher Scientific, Inc.), and maintained at $37^{\circ} \mathrm{C}$ in a humidified atmosphere containing $5 \% \mathrm{CO}_{2}$.

Cell transfection. Short hairpin (sh)RNAs targeting FTX (shRNA1，5'-GCUGAUCUGUGAGCUAGCUCU-3'; shRNA2, 5'-GUGAGCUUGUACUGUUACAUC-3'; and shRNA3, 5'-GGCUUGUUCUGCUAGAUCUGU-3'), and negative control (NC) scrambled shRNA (shNC; 5'-UGUGAG AUGCAGCCUCUAC-3') were inserted into pGPU6/Neo plasmids (Shanghai GenePharma Co., Ltd.). pcDNA3.1-FTX (oe-FTX) and control pcDNA (vector) were also purchased from Shanghai GenePharma Co., Ltd.. Cells were transfected with $10 \mathrm{nM}$ plasmids using Lipofectamine ${ }^{\circledR} 2000$ (Invitrogen; Thermo Fisher Scientific, Inc.). The transfection efficiency was analyzed using reverse transcription-quantitative PCR (RT-qPCR) after $48 \mathrm{~h}$ of transfection.

$R T$ - $q P C R$. Total RNA was extracted from tissues, thyroid cancer cell lines (FTC-236, SW-1736 and 8305C) and a normal human thyroid epithelial cell line (Nthy-ori3-1) using TRIzol ${ }^{\circledR}$ reagent (Invitrogen; Thermo Fisher Scientific, Inc.). Total RNA was reverse transcribed into cDNA using a PrimeScript RT reagent kit (Takara Bio, Inc.) according to the manufacturer's instructions. qPCR was subsequently performed using SYBR ${ }^{\circledR}$ Premix Ex Taq ${ }^{\mathrm{TM}}$ reagent (Takara Bio, Inc.). The following primer sequences were used for the qPCR: FTX forward,
5'-GTGTCTCTCTCTCTCTCTCTCTT-3' and reverse, 5'-CCT CTTCAGCAGTAGCATAGTT-3'; TGF- $\beta 1$ forward, $5^{\prime}-\mathrm{GGA}$ CATCAACGGGTTCACTA-3' and reverse, 5'-GCCATGAGA AGCAGGAAAG-3'; and GAPDH forward, 5'-ATTCCATGG CACCGTCAAGGCTGA-3' and reverse, 5'-TTCTCCATG GTGGTGAAGACGCCA-3'. The thermocycling conditions were as follows: Pre-denaturation at $95^{\circ} \mathrm{C}$ for $1 \mathrm{~min}$, followed by 40 cycles of $95^{\circ} \mathrm{C}$ for $15 \mathrm{sec}, 60^{\circ} \mathrm{C}$ for $30 \mathrm{sec}$ and $72^{\circ} \mathrm{C}$ for $30 \mathrm{sec}$. The expression levels were quantified using the $2^{-\Delta \Delta \mathrm{Cq}}$ method (19) and normalized to GAPDH expression levels.

Cell counting kit-8 (CCK-8) assay. Cell proliferation was examined using a Cell Counting kit-8 (CCK-8) assay (Thermo Fisher Scientific, Inc.) according to the manufacturer's instructions. The transfected cells were seeded into 96 -well plates at a density of $1 \times 10^{4}$ cells/well and cultured at $37^{\circ} \mathrm{C}$ with $5 \% \mathrm{CO}_{2}$ for $0,24,48$ or $72 \mathrm{~h}$. Following the incubation, $10 \mu \mathrm{l} \mathrm{CCK}-8$ reagent (Dojindo Molecular Laboratories, Inc.) was added/well. The absorbance of each well was measured at a wavelength of $450 \mathrm{~nm}$ using a microplate reader (Olympus Corporation).

Cell migration and invasion assays. Transwell chambers (8.0- $\mu \mathrm{m}$ pore size; EMD Millipore) precoated with Matrigel (invasion) or without Matrigel (migration) were used. A total of $5 \times 10^{4}$ transfected cells were plated into the upper chambers of Transwell plates in serum-free DMEM (Gibco; Thermo Fisher Scientific, Inc.), while DMEM supplemented with $20 \%$ FBS (Gibco; Thermo Fisher Scientific, Inc.) was added into the lower chambers. Following $24 \mathrm{~h}$ of incubation, cells were fixed with $4 \%$ paraformaldehyde for $20 \mathrm{~min}$ at room temperature and stained with $0.1 \%$ crystal violet for $20 \mathrm{~min}$ at room temperature. The migratory and invasive cells were counted in five randomly selected fields of view using a light microscope (magnification, x200; Zeiss $\mathrm{GmbH}$ ). All experiments were performed 3 times.

TUNEL assay. A TUNEL assay was used to analyze cell apoptosis. Briefly, cells were fixed with $4 \%$ paraformaldehyde for $1 \mathrm{~h}$ at $4^{\circ} \mathrm{C}$ and permeabilized with $0.1 \%$ Triton $\mathrm{X}-100$ following treatment. TUNEL assay solution (Invitrogen; Thermo Fisher Scientific, Inc.) was subsequently incubated with the cells for $1 \mathrm{~h}$ at $37^{\circ} \mathrm{C}$. Next, the TUNEL-stained cells were counterstained with DAPI $(2 \mu \mathrm{g} / \mathrm{ml}$; Beyotime Institute of Biotechnology) under antifade mounting medium for $15 \mathrm{~min}$ at room temperature. Images were acquired from five randomly selected fields of view using a fluorescence microscope (magnification, x200). TUNEL-positive cells were counted using a cell counter (BD Biosciences).

Cell cycle distribution assay. A total of $1 \times 10^{4}$ transfected FTC-236 and $8305 \mathrm{C}$ cells were fixed with $75 \%$ ethanol at $4^{\circ} \mathrm{C}$ for $12 \mathrm{~h}$ and washed with PBS twice. Cells were subsequently resuspended in staining buffer containing $450 \mu \mathrm{l}$ PI and $50 \mu \mathrm{l}$ RNaseA in the dark for $30 \mathrm{~min}$ at room temperature. Cell cycle distribution was analyzed using a FACSCalibur flow cytometer (BD Biosciences). Cell cycle analysis was conducted using ModFit 2.0 software (BD Biosciences).

Xenograft tumor model. A total of 6 male nude mice (age, 4-6 weeks; weight, $20 \mathrm{~g}$ ) were obtained for the xenograft 

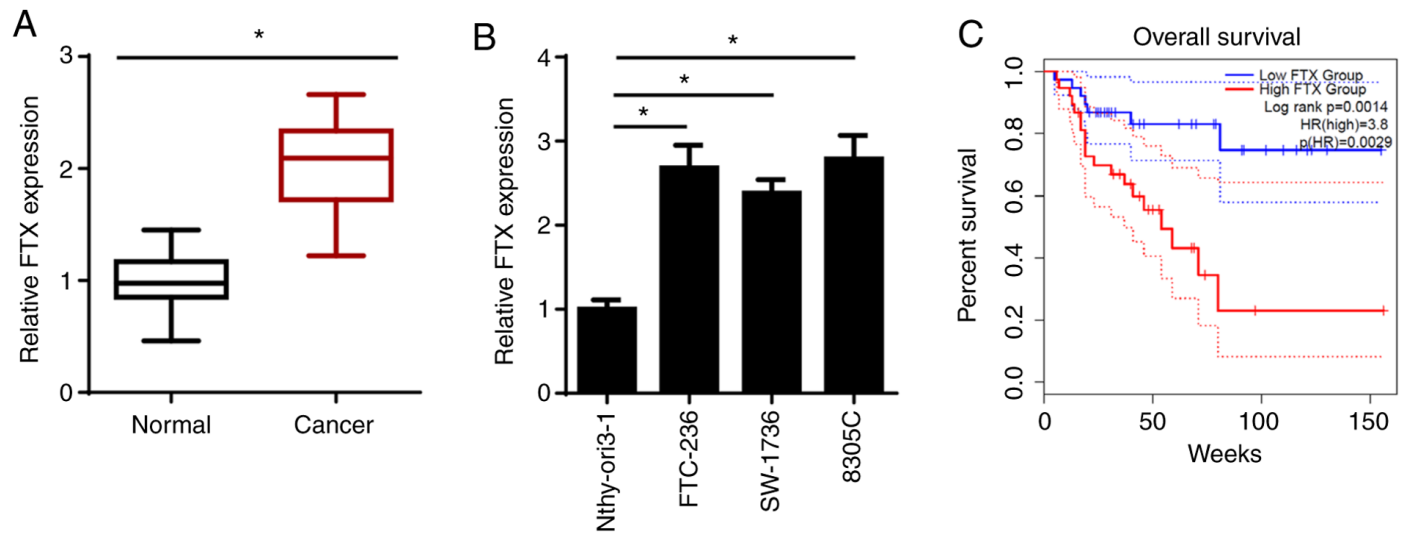

Figure 1. FTX expression is elevated in thyroid cancer tissues and cell lines, and high FTX expression is associated with an undesirable prognosis. (A) FTX expression was detected in 58 thyroid cancer tissues and matched adjacent normal tissues. (B) The relative FTX expression in thyroid cancer cell lines (FTC-236, SW-1736 and 8305C) and a normal human thyroid epithelial cell line (Nthy-ori3-1) was determined by reverse transcription-quantitative PCR. (C) Survival plot of thyroid cancer patients with high or low FTX expression, as analyzed by the Kaplan-Maier method. "P<0.05. FTX, FTX transcript X inactive specific transcript regulator.

assays. The mice were maintained under specific pathogen-free conditions, with free access to water and food, at a room temperature of $26-28^{\circ} \mathrm{C}$, humidity of $60 \pm 10 \%$ and under a 12 -h light/dark cycle. A total of $100 \mu 1$ PBS containing FTC-236 cells $\left(5 \times 10^{6}\right)$ transfected with shNC or shRNA1 was injected into the back of each mouse. The volume of the xenograft tumors was measured every 7 days. After 28 days, all mice were euthanized by cervical dislocation after deep anesthesia with isoflurane ( $2 \%$ for induction and maintenance; Baxter Healthcare Corporation), and the xenograft tumors were excised and weighed. The tumor volume was calculated using the following formula: $\mathrm{V}=1 / 2 \times \mathrm{L} \mathrm{x} \mathrm{W}{ }^{2}$. The maximum tumor diameter was $15 \mathrm{~mm}$. Animal experimental protocols were approved by the Animal Welfare Committee of Anhui No. 2 Provincial People's Hospital, and all experimental procedures were performed according to the guidelines from the National Institutes of Health.

Immunohistochemistry (IHC). The tissue expression of TGF- $\beta 1$ was evaluated via IHC based on the intensity and the proportion of positively stained cells, as previously described (19). Tumor tissues were fixed with $10 \%$ paraformaldehyde at room temperature for $12 \mathrm{~h}$, embedded in paraffin, and sliced into $4-\mu \mathrm{m}$ thick sections. Sections were blocked with immunol staining blocking buffer (cat. no. P0102; Beyotime Institute of Biotechnology) at room temperature for $1 \mathrm{~h}$ and then incubated with primary antibody against TGF- $\beta 1$ (1:1,000; cat. no. ab215715; Abcam) overnight at $4^{\circ} \mathrm{C}$, followed by incubation with secondary antibody $(1: 2,000$; cat. no. ab205718; Abcam) at room temperature for $20 \mathrm{~min}$. The sections were stained with 3,3'-diaminobenzidine and counterstained with hematoxylin for $5 \mathrm{~min}$ at room temperature. Images were captured under a light microscope (Olympus Corporation; magnification, $\mathrm{x} 200$ ).

Western blotting. Total protein was extracted from thyroid cancer cells (TC-236 and 8305C) using RIPA lysis buffer (Beyotime Institute of Biotechnology) at $48 \mathrm{~h}$ post transfection. Protein concentrations were determined using a BCA protein assay kit (Beyotime Institute of Biotechnology). The protein $(10 \mu \mathrm{g} /$ lane) was quantified and separated on a $10 \%$ gel via SDS-PAGE. The separated proteins were subsequently transferred onto polyvinylidene fluoride membranes (Bio-Rad Laboratories, Inc.) and blocked with 5\% skimmed milk for $2 \mathrm{~h}$ at room temperature. The membranes were then incubated with the following primary antibodies overnight at $4^{\circ} \mathrm{C}$ : Anti-Bcl-2 (1:1,000; cat. no. ab32124), anti-Bax (1:1,000; cat. no. ab32503), anti-TGF- $\beta 1$ (1:1,000; cat. no. ab215715) and anti-GAPDH (1:1,000; cat. no. ab8245) (all Abcam). Following the primary antibody incubation, the membranes were incubated with horseradish peroxidase-conjugated secondary antibody (1:1,000; cat. nos. ab205719 and ab205718; Abcam) for $1 \mathrm{~h}$ at room temperature. Protein bands were visualized using an ECL reagent (Cytiva).

Statistical analysis. Statistical analyses were performed using GraphPad Prism 6.0 (GraphPad Software, Inc.). Data are presented as the mean $\pm \mathrm{SD}$ of three independent experiments. A $\chi^{2}$ test was used to determine the association between the expression levels of FTX and the clinicopathological features of the patients. Statistical differences between thyroid cancer and adjacent normal tissues were analyzed using a paired Student's t-test, while the differences between experiment and control groups were analyzed using an unpaired Student's t-test. A one-way ANOVA followed by Tukey's post hoc test was used to determine statistical differences among multiple groups. The overall survival rate was analyzed using the Kaplan-Meier method and a log-rank test. The patients were divided into high and low expression groups based on the mean value of FTX expression in patients with thyroid cancer. $\mathrm{P}<0.05$ was considered to indicate a statistically significant difference.

\section{Results}

FTX expression levels are upregulated in thyroid cancer tissues and cell lines, and upregulated FTX expression is associated with an unfavorable prognosis. To determine the biological functions of FTX, the expression levels of FTX were analyzed in thyroid cancer and adjacent normal tissues using RT-qPCR. As shown in Fig. 1A, FTX expression levels were 
significantly upregulated in thyroid cancer tissues compared with those in adjacent normal tissues. Similarly, the expression levels of FTX were significantly upregulated in thyroid cancer cell lines (FTC-236; differentiated thyroid carcinoma, SW-1736; anaplastic thyroid carcinoma, and 8305C; anaplastic thyroid carcinoma) compared with those in the normal human thyroid epithelial cell line, Nthy-ori3-1. FTC-236 and 8305C cell lines were chosen for use in subsequent experiments, as FTX expression levels were the highest in these two cell lines (Fig. 1B). To determine the association between FTX expression levels and the prognosis of patients with thyroid cancer, Kaplan-Meier analysis was performed. As shown in Fig. 1C, patients with thyroid cancer and high FTX expression levels exhibited a lower overall survival rate compared with those patients with low expression levels. In addition, FTX expression levels were associated with the clinical stage and lymph node metastasis, but not with sex, age, pathological type or tumor size (Table I). These results suggested that FTX expression levels may be upregulated in thyroid cancer and associated with a poor prognosis in patients with this disease.

Knockdown of FTX inhibits proliferation and migration, and induces apoptosis in thyroid cancer cells. To determine the biological functions of FTX in thyroid cancer cells, FTX expression levels were knocked down in FTC-236 and 8305C cells using three shRNAs. Due to the greatest knockdown efficiency, shRNA1 and shRNA3 were selected for use in subsequent experiments (Fig. 2A). The proliferation rates of FTC-236 and $8305 \mathrm{C}$ cells transfected with shRNA1 and shRNA3 were analyzed using a CCK-8 assay; the results revealed that FTX knockdown significantly decreased cell proliferation in the thyroid cancer cells (Fig. 2B). In addition, the results of the Transwell assays demonstrated that the knockdown of FTX attenuated the migratory and invasive abilities of the thyroid cancer cells (Fig. 2C and D). Moreover, according to the results of the TUNEL assay, FTX knockdown also alleviated the apoptosis of the thyroid cancer cells (Fig. 2E). Western blot analysis revealed that the knockdown of FTX upregulated the protein expression levels of Bax and downregulated Bcl-2 expression levels in the thyroid cancer cells (Fig. 2F). In addition, flow cytometric analysis found that FTX knockdown initiated cell cycle arrest in the $G_{0} / G_{1}$ phase (Fig. 2G). These data indicated that FTX knockdown may repress the progression of thyroid cancer.

Overexpression of FTX accelerates proliferation, migration and invasion, and alleviates apoptosis in thyroid cancer cells. The effect of the overexpression of FTX on thyroid cancer cells was investigated by transfecting FTC-236 and 8305C cells with vector or oe-FTX, and the transfection efficiency was verified using RT-qPCR (Fig. 3A). The results of the cell proliferation assay showed that FTX overexpression promoted the proliferation of the thyroid cancer cells (Fig. 3B). In addition, the results of the Transwell assays demonstrated that the overexpression of FTX expression markedly enhanced the migration and invasion of the thyroid cancer cells (Fig. 3C and D). Furthermore, the overexpression of FTX attenuated the apoptosis of thyroid cancer cells (Fig. 3E). In addition, the overexpression of FTX also significantly increased cell proliferation, migration and invasion, but inhibited the apoptosis of Nthy-ori3-1
Table I. Clinical characteristics and FTX expression of patients with thyroid cancer.

\begin{tabular}{lccc}
\hline & \multicolumn{2}{c}{ FTX expression } & \\
\cline { 2 - 3 } Variable & Low, $\mathrm{n}$ & High, $\mathrm{n}$ & P-value \\
\hline All cases & 23 & 35 & \\
Age, years & & & \\
$\quad<50$ & 12 & 17 & $>0.05$ \\
$\geq 50$ & 11 & 18 & \\
Sex & & & \\
Male & 10 & 15 & $>0.05$ \\
Female & 13 & 20 & \\
Pathological type & & & \\
Papillary adenocarcinoma & 9 & 11 & $>0.05$ \\
Follicular adenocarcinoma & 14 & 24 & \\
Lymph node metastasis & & & \\
Yes & 18 & 23 & $<0.05$ \\
No & 5 & 12 & \\
Tumor size, cm & & & \\
$\quad<3$ & 11 & 16 & $>0.05$ \\
$\geq 3$ & 12 & 19 & \\
Tumor stage & & & \\
I/II & 10 & 25 & \\
III/IV & & 10 & \\
\hline
\end{tabular}

FTX, FTX transcript X inactive specific transcript regulator.

cells (Fig. 3F-I). These results indicated that the overexpression of FTX may promote the proliferation, migration and invasion, but attenuate the apoptosis of thyroid cancer cells.

Knockdown of FTX represses thyroid cancer growth in vivo. Due to the observed inhibitory effect of FTX knockdown on thyroid cancer cell proliferation, the effect of FTX knockdown on tumor growth in vivo was further analyzed using xenograft models. The data indicated that xenograft tumors derived from FTC-236 cells stably transfected with shRNA1 grew significantly more slowly compared with those of the shNC group (Fig. 4A), and the tumor volume and weight were markedly decreased in the shRNA1 group compared with the shNC group (Fig. 4B and C). These findings suggested that the knockdown of FTX may suppress the growth of thyroid cancer in vivo.

FTX positively regulates TGF- $\beta 1$ expression levels in thyroid cancer cells. It was previously reported that the TGF- $\beta$ signaling pathway played an important role in promoting tumor metastasis $(20,21)$. Moreover, IHC showed that the expression of TGF- $\beta 1$ in thyroid cancer tissues was higher compared with that in adjacent normal tissues (Fig. 5A). Therefore, in the present study, it was hypothesized that FTX may promote the development of thyroid cancer by modulating TGF- $\beta 1$ expression. To validate this hypothesis, the expression levels of TGF- $\beta 1$ in FTC-236 and 8305C cells 

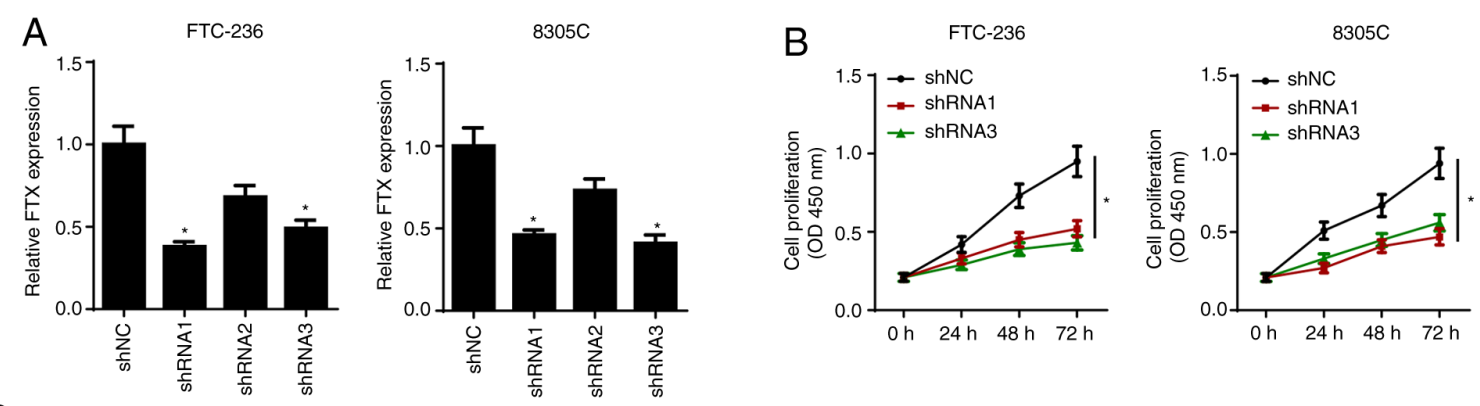

C
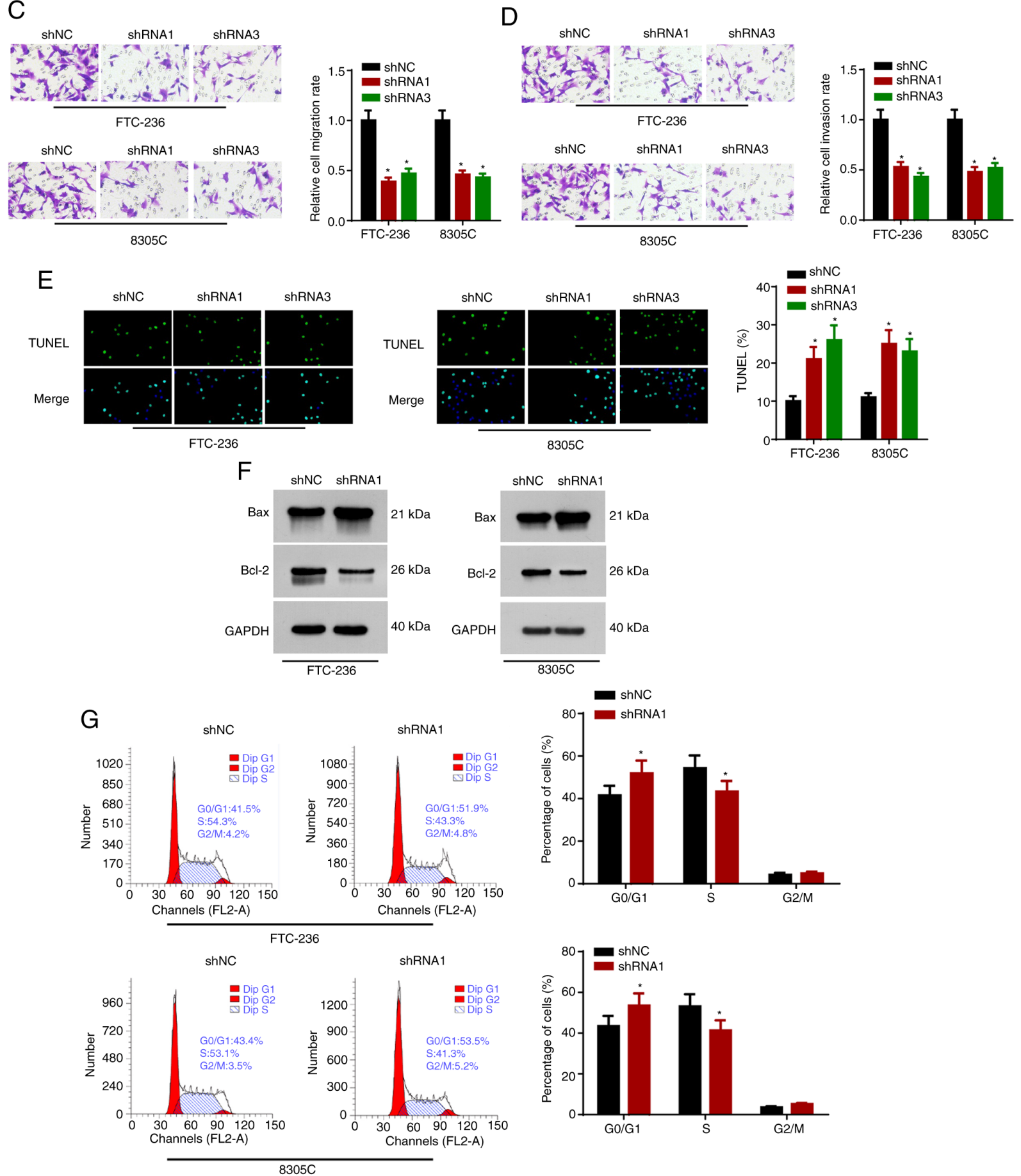

Figure 2. FTX depletion inhibits proliferative and metastatic capacities, and induces apoptosis of thyroid cancer cells. FTC-236 and $8305 \mathrm{C}$ cells were transfected with shNC, shRNA1, shRNA2 and shRNA3, respectively. (A) The transfection efficacy of shNC, shRNA1, shRNA2 and shRNA3 was detected by reverse transcription-quantitative PCR. (B) Proliferation of FTC-236 and 8305C cells was measured by CCK-8 assay at 0, 24, 48 and $72 \mathrm{~h}$. (C and D) Transwell assay exhibited the migration and invasion of FTC-236 and 8305C cells (magnification, x200). (E) Apoptosis of FTC-236 and 8305C cells was analyzed by TUNEL assay (magnification, x200). (F) Protein expression levels of Bax and Bcl-2 in thyroid cancer cells transfected with sh-NC or shRNA1 were detected by western blot analysis. (G) The cell cycle of transfected FTC-236 and $8305 \mathrm{C}$ cells was assessed by flow cytometry analysis. ${ }^{*} \mathrm{P}<0.05$ vs. shNC. FTX, FTX transcript X inactive specific transcript regulator; sh, short hairpin; NC, negative control. 

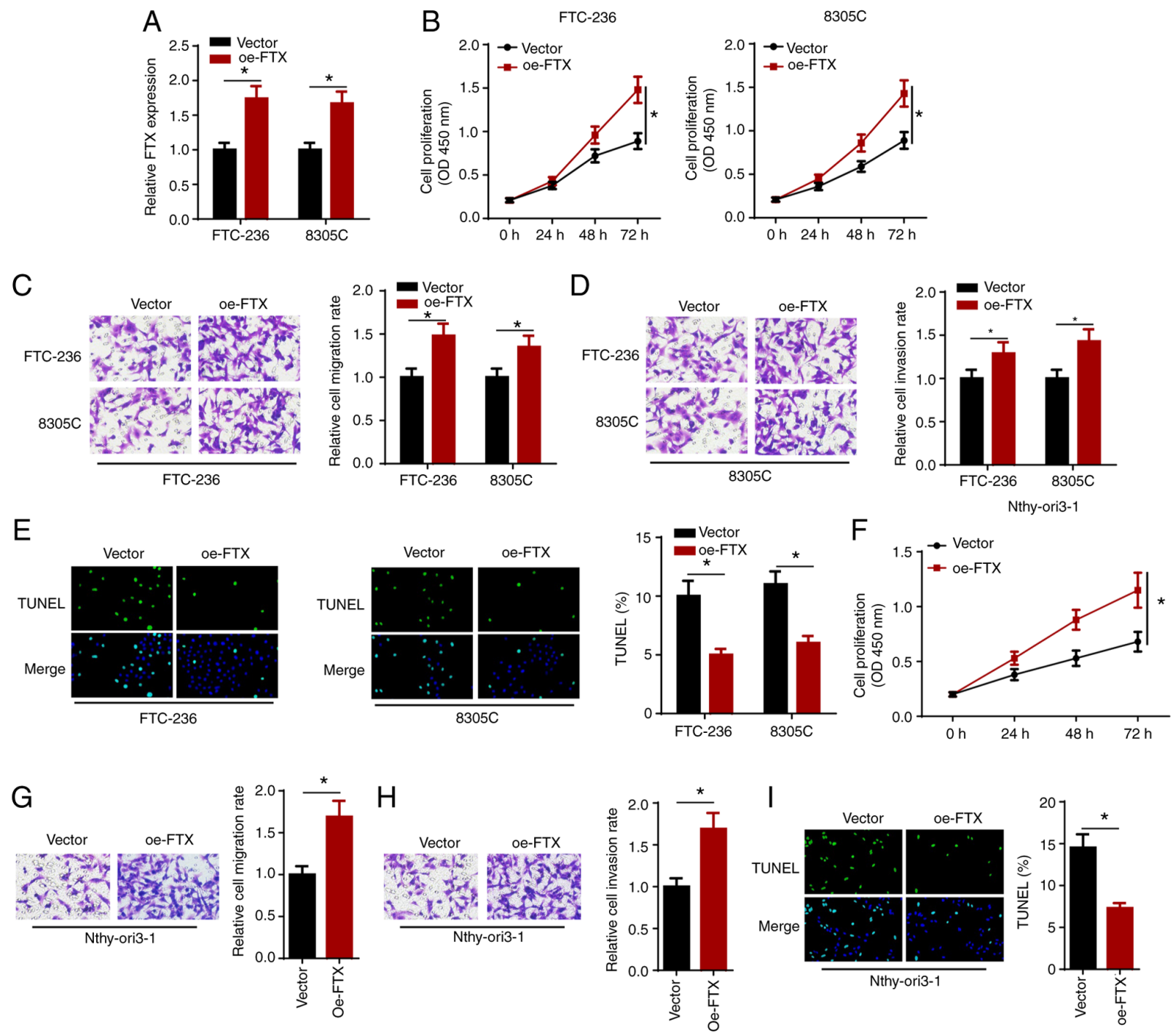

Figure 3. FTX overexpression accelerates proliferation, migration and invasion, and alleviates apoptosis of thyroid cancer cells. FTC-236 and $8305 \mathrm{C}$ cells were transfected with vector and oe-FTX, respectively. (A) The transfection efficacy of vector and oe-FTX was detected by reverse transcription-quantitative PCR. (B) Viability of FTC-236 and 8305C cells was measured by CCK-8 assay at 0,24, 48 and $72 \mathrm{~h}$. (C and D) Transwell assay exhibited the migration and invasion of FTC-236 and 8305C cells (magnification, x200). (E) Apoptosis of FTC-236 and 8305C cells was analyzed by TUNEL assay (magnification, x200). (F-I) The cell viability, migration, invasion and apoptosis (magnification, x200) of Nthy-ori3-1 cells transfected with FTX overexpression plasmid were analyzed by functional assays. "P<0.05. FTX, FTX transcript X inactive specific transcript regulator; oe, overexpression.
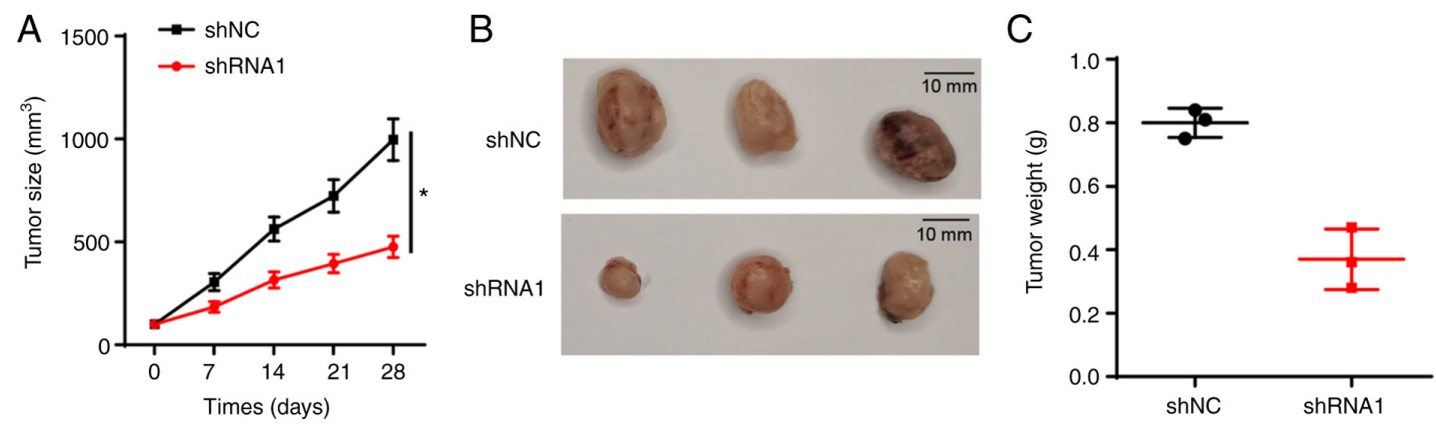

Figure 4. FTX silencing represses tumor growth of thyroid cancer in vivo. FTC-236 cells transfected with shNC or shRNA1 were adopted to establish xenograft tumor models. (A) Tumor volume was calculated every 7 days and growth curves of tumors were plotted. (B and C) After 28 days, mice were sacrificed to obtain resected tumors, and tumor weight in each group was examined. "P<0.05. FTX, FTX transcript X inactive specific transcript regulator; sh, short hairpin; $\mathrm{NC}$, negative control.

transfected with shNC or shRNA1 were analyzed. As shown in Fig. 5B and C, RT-qPCR and western blot analysis revealed that the knockdown of FTX downregulated the expression levels of TGF- $\beta 1$. These results suggested that FTX may 

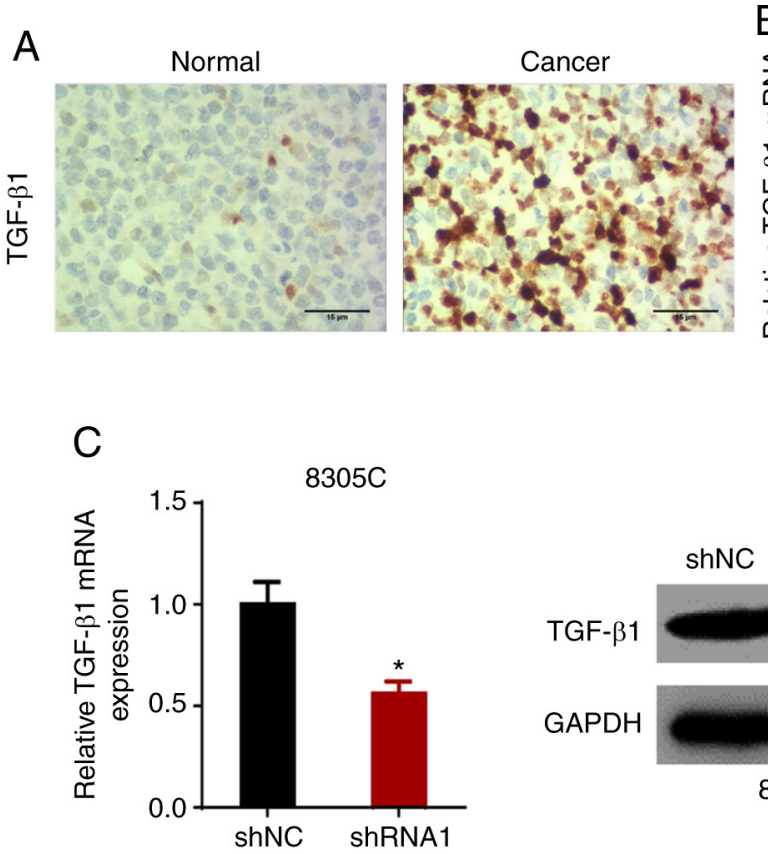
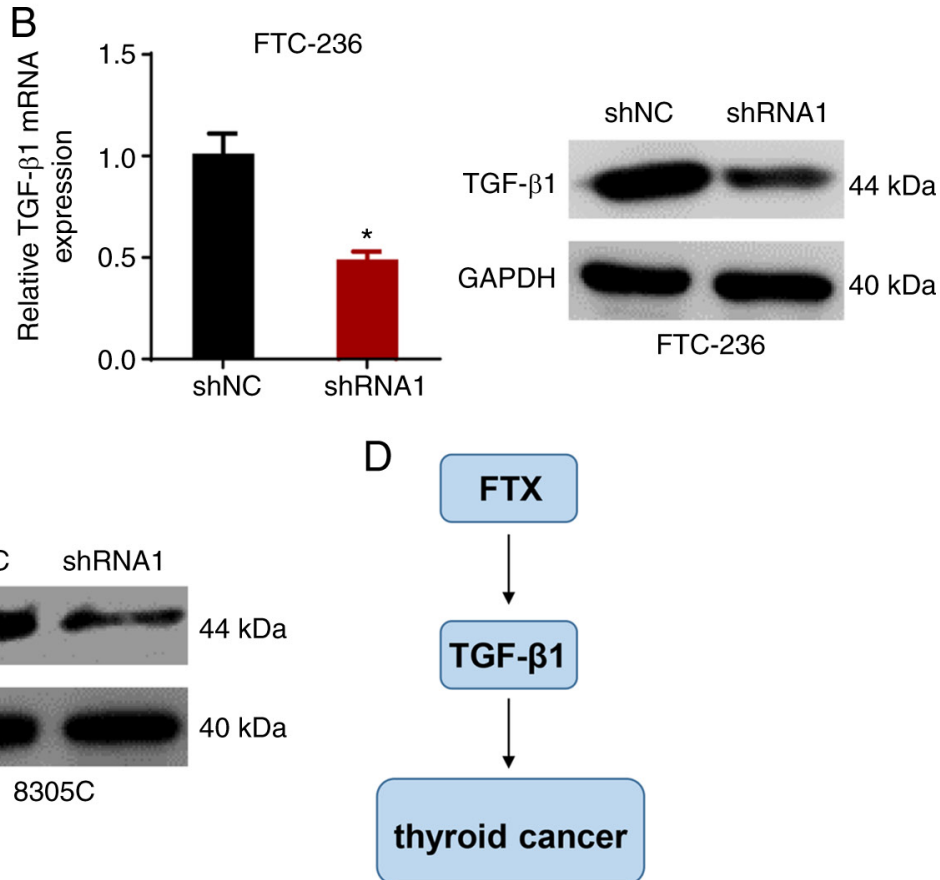

Figure 5. FTX positively regulates TGF- $\beta 1$ level in thyroid cancer cells. FTC-236 and 8305C cells were transfected with shNC and shRNA1, respectively. (A) IHC showed that the expression of TGF- $\beta 1$ in thyroid cancer tissues and adjacent normal tissues (scale bar, $15 \mu \mathrm{m}$ ). (B and C) Reverse transcription-quantitative PCR and western blot analysis showed the level of TGF- $\beta 1$ expression in FTC-236 and 8305C cells transfected with shNC and shRNA1, respectively. (D) Schematic diagram of proposed mechanism. "P<0.05. FTX, FTX transcript X inactive specific transcript regulator; sh, short hairpin; NC, negative control.

positively regulate TGF- $\beta 1$ expression levels in thyroid cancer cells (Fig. 5D).

\section{Discussion}

Although significant advances have been made in the screening, diagnosis and treatment of thyroid cancer, the majority of patients with thyroid cancer have an undesirable prognosis. Numerous previous studies have attempted to further understand the underlying molecular mechanisms of thyroid cancer; however, to the best of our knowledge, the pathogenesis of thyroid cancer remains unclear.

The dysregulated expression of IncRNAs has been reported to be involved in multiple cellular activities, including oncogenesis $(22,23)$. In addition, a large number of lncRNAs have been recognized as potential sensitive biomarkers for thyroid cancer in the clinic. For example, lncRNA ST binding factor 2-antisense RNA 1 (AS1) accelerated the growth of papillary thyroid cancer by modulating the miR-431-5p/ CDK14 axis (24). The overexpression of opa interacting protein 5-AS1 promoted thyroid cancer progression and predicted an unfavorable prognosis (25). In addition, lncRNA aspartyl-tRNA synthetase 1-AS1 contributed to thyroid cancer development by interacting with miR-129 (26). Therefore, it is has been widely suggested that lncRNAs may function as biomarkers for thyroid cancer diagnosis and prognosis, and may represent novel targets for thyroid cancer treatment.

FTX has been reported to play a role in various types of cancer. In lung adenocarcinoma, the expression levels of FTX were upregulated and promoted the progression of the cell lines (27). In addition, FTX targeted miR-342-3p in glioma to accelerate tumor growth and metastasis (28). Another previous study reported that the knockdown of FTX suppressed the progression of renal cell carcinoma (29). Moreover, FTX overexpression promoted tumorigenesis in osteosarcoma by targeting the miR-214-5p/SOX4 signaling axis (30). Recently, Luzón-Toro et al (18) reported that FTX was significantly upregulated in sporadic medullary thyroid cancer. The findings of the present study revealed that the expression levels of FTX were upregulated in thyroid cancer tissues and cells, and high FTX expression was associated with lymph node metastasis and clinical stage. Moreover, the upregulated expression levels of FTX favored a poor prognosis in patients with thyroid cancer and serum FTX had a relatively high diagnostic value. Results of the functional analysis demonstrated that the knockdown of FTX significantly inhibited proliferation, invasion, migration and cell cycle progression, and induced apoptosis in thyroid cancer cells. In addition, the suppressive effects of FTX knockdown on thyroid cancer progression were also confirmed using an in vivo xenograft tumor assay. These results indicated that FTX may promote the progression of thyroid cancer and may represent a novel biomarker for the treatment and prognosis of thyroid cancer.

An increasing number of studies have reported that TGF- $\beta 1$ contributes to the progression of various cancer types, including thyroid cancer. For example, forkhead box D3-AS1 promoted the aggressive biological behaviors of thyroid cancer by activating the TGF- $\beta 1 /$ Smads signaling pathway (31). In addition, miR-483 targeted par-3 family cell polarity factor to enhance TGF- $\beta 1$-induced migration and invasion in thyroid cancer cells (32). To determine whether FTX exerted its biological functions by regulating TGF- $\beta 1$ in the present study, RT-qPCR and western blotting were performed; the results revealed that the knockdown of FTX downregulated the 
expression levels of TGF- $\beta 1$. These data indicated that FTX may regulate thyroid cancer progression via TGF- $\beta 1$.

However, there are several limitations to the present study. First, the study only investigated the effect of FTX on thyroid cancer. Therefore, the downstream targets or signaling pathways of FTX should be further investigated in future studies. Second, only a small number of mice were used for the in vivo studies. Therefore, an increased number of mice should be used in the in vivo xenograft model studies in the future to improve the reliability of the experiments.

In conclusion, the findings of the present study suggested that FTX may exert an important role in thyroid cancer progression; therefore, FTX may represent a novel biomarker for the diagnosis, treatment and prognosis of thyroid cancer.

\section{Acknowledgements}

Not applicable.

\section{Funding}

No funding was received.

\section{Availability of data and materials}

The datasets used and/or analyzed during the current study are available from the corresponding author on reasonable request.

\section{Authors' contributions}

PW and HJ designed the present study. YZ and WW performed the experiments. PW, YZ and WW analyzed the data and prepared the figures. $\mathrm{PW}$ and $\mathrm{HJ}$ drafted the initial manuscript, reviewed and revised the manuscript. All authors have read and approved the manuscript. PW and HJ confirm the authenticity of all the raw data.

\section{Ethics approval and consent to participate}

Each patient provided written informed consent prior to participation in the study. The present study was approved by the Ethical Committee of Anhui No. 2 Provincial People's Hospital.

\section{Patient consent for publication}

Not applicable.

\section{Competing interests}

The authors declare that they have no competing interests.

\section{References}

1. Segev DL, Umbricht C and Zeiger MA: Molecular pathogenesis of thyroid cancer. Surg Oncol 12: 69-90, 2003.

2. Ferlay J,SoerjomataramI, Siegel RL, Torre LA and Jemal A: Global cancer statistics 2018: GLOBOCAN estimates of incidence and mortality worldwide for 36 cancers in 185 countries. CA Cancer J Clin 68: 394-424, 2018

3. Sipos JA and Mazzaferri EL: Thyroid cancer epidemiology and prognostic variables. Clin Oncol (R Coll Radiol) 22: 395-404, 2010.
4. Janovitz T and Barletta JA: Clinically relevant prognostic parameters in differentiated thyroid carcinoma. Endocr Pathol 29: 357-364, 2018.

5. Thompson LD, Wieneke JA, Paal E, Frommelt RA, Adair CF and Heffess CS: A clinicopathologic study of minimally invasive follicular carcinoma of the thyroid gland with a review of the English literature. Cancer 91: 505-24, 2001.

6. Federico C, Sun J, Muz B, Alhallak K, Cosper PF, Muhammad N, Jeske A, Hinger A, Markovina S, Grigsby P, et al: Localized Delivery of Cisplatin to Cervical cancer improves its therapeutic efficacy and minimizes its side effect profile. Int J Radiat Oncol Biol Phys 109: 1483-1494, 2021.

7. Mohammad N, Singh SV, Malvi P, Chaube B, Athavale D, Vanuopadath M, Nair SS, Nair B and Bhat MK: Strategy to enhance efficacy of doxorubicin in solid tumor cells by methyl- $\beta$-cyclodextrin: Involvement of p53 and Fas receptor ligand complex. Sci Rep 5: 11853, 2015.

8. Muhammad N, Bhattacharya S, Steele R and Ray RB: Anti-miR-203 suppresses ER-positive breast cancer growth and stemness by targeting SOCS3. Oncotarget 7: 58595-58605, 2016.

9. Muhammad N, Bhattacharya S, Steele R, Phillips N and Ray RB: Involvement of c-Fos in the promotion of cancer stem-like cell properties in head and neck squamous cell carcinoma. Clin Cancer Res 23: 3120-3128, 2017.

10. Liu H, Deng H, Zhao Y, Li C and Liang Y: LncRNA XIST/ miR-34a axis modulates the cell proliferation and tumor growth of thyroid cancer through MET-PI3K-AKT signaling. J Exp Clin Cancer Res 37: 279, 2018.

11. Huang JK, Ma L, Song WH, Lu BY, Huang YB, Dong HM, Ma XK, Zhu ZZ and Zhou R: LncRNA-MALAT1 promotes angiogenesis of thyroid cancer by modulating tumor-associated macrophage FGF2 protein secretion. J Cell Biochem 118: 4821-4830, 2017.

12. Qin Y, Xue B, Liu C, Wang X, Tian R, Xie Q, Guo M, Li G, Yang D and Zhu H: NLRX1 mediates MAVS degradation to attenuate the hepatitis $C$ virus-induced innate immune response through PCBP2. J Virol 91: e01264-17, 2017.

13. Li B, Ren P and Wang Z: Long non-coding RNA Ftx promotes osteosarcoma progression via the epithelial to mesenchymal transition mechanism and is associated with poor prognosis in patients with osteosarcoma. Int J Clin Exp Pathol 11: 4503-4511, 2018.

14. Zhang F, Wang XS, Tang B, Li PA, Wen Y and Yu PW: Long non-coding RNA FTX promotes gastric cancer progression by targeting miR-215. Eur Rev Med Pharmacol Sci 24: 3037-3048, 2020.

15. Yang Y, Zhang J, Chen X, Xu X, Cao G, Li H and Wu T: LncRNA FTX sponges miR-215 and inhibits phosphorylation of vimentin for promoting colorectal cancer progression. Gene Ther 25: 321-330, 2018.

16. Liang $\mathrm{Y}$ and Lu H: Long noncoding RNA FTX is associated with prognosis of glioma patients. J Gene Med 22: e3237, 2020.

17. Jiang W, Zhang B, Sun J, Liu Y, Bi Y and Wei H: LncRNA FTX promotes the tumorigenesis of lung adenocarcinoma by targeting miR-300. Panminerva Med: Jan 24, 2020 (Epub ahead of print). doi: 10.23736/S0031-0808.19.03823-0.

18. Luzón-Toro B, Villalba-Benito L, Fernández RM, Torroglosa A, Antiñolo G and Borrego S: RMRP, RMST, FTX and IPW: Novel potential long non-coding RNAs in medullary thyroid cancer. Orphanet J Rare Dis 16: 4, 2021.

19. Livak KJ and Schmittgen TD: Analysis of relative gene expression data using real-time quantitative PCR and the 2(-Delta Delta C(T)) method. Methods 25: 402-408, 2001.

20. Sun DY, Wu JQ, He ZH, He MF and Sun HB: Cancer-associated fibroblast regulate proliferation and migration of prostate cancer cells through TGF- $\beta$ signaling pathway. Life Sci 235: 116791, 2019.

21. Dai G, Sun B, Gong T, Pan Z, Meng Q and Ju W: Ginsenoside Rb2 inhibits epithelial-mesenchymal transition of colorectal cancer cells by suppressing TGF- $\beta /$ Smad signaling. Phytomedicine 56 : 126-135, 2019.

22. Choudhari R, Sedano MJ, Harrison AL, Subramani R, Lin KY, Ramos EI, Lakshmanaswamy R and Gadad SS: Long noncoding RNAs in cancer: From discovery to therapeutic targets. Adv Clin Chem 95: 105-147, 2020.

23. Chan JJ and Tay Y: Noncoding RNA:RNA regulatory networks in cancer. Int J Mol Sci 19: 1310, 2018.

24. Wen HL, Xu ZM, Wen D, Lin SY, Liang Y and Xie JP: Long noncoding RNAs SET-binding factor 2-antisense RNA1 promotes cell growth through targeting miR-431-5p/CDK14 axis in human papillary thyroid cancer. Kaohsiung J Med Sci 36: 808-816, 2020. 
25. Li Q, Chen W, Luo R, Zhang Z, Song M, Chen W, Yang Z, Yang Y, Guo Z and Yang A: Upregulation of OIP5-AS1 predicts poor prognosis and contributes to thyroid cancer cell proliferation and migration. Mol Ther Nucleic Acids 20: 279-291, 2020.

26. Zheng W, Tian X, Cai L, Shen YM, Cao QS, Yang JY and Tian GY: LncRNA DARS-AS1 regulates microRNA-129 to promote malignant progression of thyroid cancer. Eur Rev Med Pharmacol Sci 23: 10443-10452, 2019.

27. Huo X, Wang H, Huo B, Wang L, Yang K, Wang J, Wang L and Wang H: FTX contributes to cell proliferation and migration in lung adenocarcinoma via targeting miR-335-5p/NUCB2 axis. Cancer Cell Int 20: 89, 2020.

28. Zhang W, Bi Y, Li J, Peng F, Li H, Li C, Wang L, Ren F, Xie C, Wang $\mathrm{P}$, et al: Long noncoding RNA FTX is upregulated in gliomas and promotes proliferation and invasion of glioma cells by negatively regulating miR-342-3p. Lab Invest 97: 447-457, 2017.

29. He X, Sun F, Guo F, Wang K, Gao Y, Feng Y, Song B, Li W and Li Y: Knockdown of long noncoding RNA FTX inhibits proliferation, migration, and invasion in renal cell carcinoma cells. Oncol Res 25: 157-166, 2017.
30. Chen H, Liu T, Ouyang H, Lin S, Zhong H, Zhang H and Yang Y: Upregulation of FTX promotes osteosarcoma tumorigenesis by increasing SOX4 expression via miR-214-5p. Onco Targets Ther 13: 7125-7136, 2020.

31. Chen Y, Gao H and Li Y: Inhibition of LncRNA FOXD3-AS1 suppresses the aggressive biological behaviors of thyroid cancer via elevating miR-296-5p and inactivating TGF- $\beta 1 /$ Smads signaling pathway. Mol Cell Endocrinol 500: 110634, 2020

32. Zhang X, Liu L, Deng X, Li D, Cai H, Ma Y, Jia C, Wu B, Fan Y and Lv Z: MicroRNA 483-3p targets Pard3 to potentiate TGF- $\beta 1$-induced cell migration, invasion, and epithelial-mesenchymal transition in anaplastic thyroid cancer cells. Oncogene 38: 699-715, 2019.

This work is licensed under a Creative Commons Attribution-NonCommercial-NoDerivatives 4.0 International (CC BY-NC-ND 4.0) License. 